\title{
Recreational Nitrous Oxide Abuse-Induced Vitamin B12 Deficiency in a Patient Presenting with Hyperpigmentation of the Skin
}

\author{
Tsung-Ta Chiang $^{a} \quad$ Chih-Tsung Hung ${ }^{b}$ Wei-Ming Wang ${ }^{b, d}$ \\ Jiunn-Tay Lee ${ }^{c}$ Fu-Chi Yang ${ }^{c}$ \\ Departments of ${ }^{a}$ Internal Medicine, ${ }^{b}$ Dermatology and ${ }^{c}$ Neurology, Tri-Service \\ General Hospital, National Defense Medical Center, and ${ }^{\mathrm{d}}$ Department of Biochemistry, \\ National Defense Medical Center, Taipei, Taiwan, ROC
}

\section{Key Words}

Nitrous oxide abuse $\cdot$ Nitrous oxide intoxication · Vitamin B12 deficiency $\cdot$ Hyperpigmentation

\begin{abstract}
Vitamin B12 deficiency causes skin hyperpigmentation, subacute combined degeneration of the spinal cord, and megaloblastic anemia. Although vitamin B12 deficiency rarely occurs in well-nourished, healthy, young people, nitrous oxide $\left(\mathrm{N}_{2} \mathrm{O}\right)$ intoxication is an important cause of vitamin $\mathrm{B} 12$ deficiency in this cohort. $\mathrm{N}_{2} \mathrm{O}$, a colorless gas used as an anesthetic since the late 19th century because of its euphoric and analgesic qualities, is now used as a recreational drug and is available via the Internet and at clubs. Here, we describe the case of a 29-yearold woman presenting with skin hyperpigmentation as her only initial symptom after $\mathrm{N}_{2} \mathrm{O}$ abuse for approximately 2 years. $\mathrm{N}_{2} \mathrm{O}$ intoxication-induced vitamin $\mathrm{B} 12$ deficiency was diagnosed based on the skin pigmentation that had manifested over the dorsa of her fingers, toes, and trunk, coupled with myeloneuropathy of the posterior and lateral columns, a low serum vitamin B12 level, an elevated serum homocysteine level, and the $\mathrm{N}_{2} \mathrm{O}$ exposure revealed while establishing the patient's history. Symptoms improved significantly with vitamin B12 treatment. We recommend that dermatologists consider $\mathrm{N}_{2} \mathrm{O}$ intoxicationinduced vitamin B12 deficiency as a potential cause of skin hyperpigmentation and myeloneuropathy of the posterior and lateral columns in young, otherwise healthy patients. Failure to recognize this presentation may result in inappropriate treatment, thus affecting patients' clinical outcomes.
\end{abstract}




\section{Introduction}

Vitamin B12 (cobalamin) is necessary for DNA synthesis and methylation reactions. Its deficiency is associated with neurologic, psychiatric, hematologic, cardiovascular, and gastrointestinal manifestations [1]. Less commonly, vitamin B12 deficiency results in dermatologic manifestations, including skin hyperpigmentation, hair changes, vitiligo, and angular stomatitis [2]. Vitamin B12 deficiency is usually caused by a restrictive vegetarian diet, malnutrition, malabsorption syndromes, congenital disease, gastrectomy, or other gastrointestinal tract diseases. However, it may be also induced by the recreational use of nitrous oxide $\left(\mathrm{N}_{2} \mathrm{O}\right)$, an often forgotten and important link to B12 deficiency in young, healthy individuals. To our knowledge, cases of $\mathrm{N}_{2} \mathrm{O}$ abuse-induced vitamin $\mathrm{B} 12$ deficiency presenting with cutaneous lesions are rare. Here, we report the case of a young woman who developed diffuse hyperpigmentation of the skin after 2 years of $\mathrm{N}_{2} \mathrm{O}$ abuse.

\section{Case Report}

A 29-year-old, non-vegetarian woman was admitted to our hospital because of hyperpigmentation over the dorsa of the fingers, toes (fig. 1a, b), and trunk (fig. 2a), along with intermittent numbness in the plantar region of the foot that began 2 months before admission. The numbness and hypoesthesia were initially limited to the plantar region of the foot; subsequently, these symptoms ascended to both lower legs 2 weeks before admission. The patient noted weakness in both legs, an unsteady gait, and a tendency to fall when walking. Numbness in the fingertips was also noted. The patient's medical history was not significant, and her family history was unremarkable.

On physical examination, her conjunctivae and mucous membranes were slightly pale, whereas her tongue appeared normal. Multiple, asymptomatic, hyperpigmented macular patches were noted on the trunk (fig. 2a), and no rash or dermatitis had preceded the onset of hyperpigmentation. Neurological examination revealed weakness based on hyporeflexia in all limbs and the results of manual muscle tests (MMT 4+/5). Impaired pinprick sensations below the second thoracic dermatome, poor joint positions, and vibration sensations over both lower limbs were recorded. A broad-based ataxic gait and a positive Romberg sign were also noted. There were no atypical cerebellar signs. In addition to the observed symptoms, the patient reported mild constipation, a bitter taste in the mouth, and hair loss.

Laboratory investigations revealed normocytic anemia (hemoglobin, $10.7 \mathrm{~g} / \mathrm{dl}$ [normal range, 12.0-16.0 g/dl]; mean corpuscular volume, $94.1 \mathrm{fl}$ [normal range, 79-100 fl]), leukopenia (white blood count, $4.41 \times 10^{3} / \mu \mathrm{l}$ [normal range, $\left.4.5 \times 10^{3}-11 \times 10^{3} / \mu \mathrm{l}\right]$ ), and thrombocytopenia (platelet count, $114 \times 10^{3} / \mu \mathrm{l}$ [normal range $\left.150 \times 10^{3}-400 \times 10^{3} / \mu \mathrm{l}\right]$ ). A low vitamin B12 level (204 pg/ml [normal range 211-911 pg/ml]) and high serum homocysteine level (33.35 $\mu \mathrm{mol} / \mathrm{l}$ [normal range 3.70-13.90 $\mu \mathrm{mol} / \mathrm{l}])$ were noted. Levels of human immunodeficiency virus antibody, the autoimmune profile, and levels of folic acid and endocrine hormones were within the normal ranges. A nerve conduction velocity test revealed polyneuropathy in the lower legs. Analysis of the cerebrospinal fluid revealed normal protein levels and cell counts. Examination of skin biopsy specimens showed basal layer hyperpigmentation with melanin incontinence and focal mild acanthosis.

We reevaluated the patient's medical history, taking into account the possibility of a vitamin B12 deficiency. The patient reported that she had started using $\mathrm{N}_{2} \mathrm{O}$ at parties in recent years. The frequency of $\mathrm{N}_{2} \mathrm{O}$ use was half a whipped cream propellant cartridge (approximately 7.0 liters of $\mathrm{N}_{2} \mathrm{O}$ may be released from each cartridge) daily for the last 2 
years, and 1 month before admission, this had increased to 1 propellant cartridge daily. The patient received intramuscular injections of 1,000 $\mu \mathrm{g}$ vitamin B12 daily for 1 week and then weekly for 1 month. After 4 weeks of treatment, the pigmented lesions showed clinically significant improvement (fig. 1c, d, fig. 2b).

\section{Discussion}

Vitamin B12 deficiency is associated with cutaneous, neurologic, psychiatric, hematologic, cardiovascular, cutaneous, psychomotor, and gastrointestinal manifestations [1]. Many factors can contribute to vitamin B12 deficiency, including a restrictive vegetarian diet, malnutrition, malabsorption syndromes, congenital disease (i.e. transporter defect), postgastrectomy malabsorption, pernicious anemia, and other gastrointestinal tract diseases (i.e. ileal resection, bacterial overgrowth, and inflammatory bowel disease) [1]. Furthermore, and as seen in our patient, long-term exposure to $\mathrm{N}_{2} \mathrm{O}$ can induce vitamin $\mathrm{B} 12$ deficiency. $\mathrm{N}_{2} \mathrm{O}$, a commonly used inhaled anesthetic agent, is not commonly used as a recreational drug [3]. However, $\mathrm{N}_{2} \mathrm{O}$ is available via the Internet and on the street in the form of whipped cream canisters, tanks, or in small cartridges called 'whippets'. The toxicity of $\mathrm{N}_{2} \mathrm{O}$ is based on its interaction with vitamin B12 [4] and was first reported by Banks et al. in 1968 [5]. Once $\mathrm{N}_{2} \mathrm{O}$ is cleaved into free nitrogen and oxygen, it rapidly oxidizes and irreversibly inactivates vitamin B12.

Vitamin B12 plays a critical role in methylation reactions and DNA synthesis. It is a cofactor for 2 important enzymes in humans: methionine synthase and methylmalonylcoenzyme A ( $\mathrm{CoA})$ mutase. In the cytoplasm, methionine synthase requires the reduced or Co(I) form of vitamin B12, namely, methylcobalamin, to convert homocysteine to methionine by the transfer of a methyl group from 5-methyltetrahydrofolate. This step retains the methyl donor S-adenosylmethionine for further methylation processes in the methylation cycle and links to the DNA synthesis pathway via the generation of tetrahydrofolate from 5methyltetrahydrofolate.

In mitochondria, the oxidized or $\mathrm{Co}(\mathrm{III})$ form of vitamin $\mathrm{B} 12$, namely, $5^{\prime}$ deoxyadenosylcobalamin, is required for the mitochondrial enzyme methylmalonyl-CoA mutase to convert methylmalonyl-CoA to succinyl-CoA, which is important in hemoglobin synthesis, the oxidation of odd-chain fatty acids, and the catabolism of ketogenic amino acids $[6,7]$. The reduced form of vitamin B12, as noted previously, is an important cofactor for methionine synthase, a key enzyme in DNA synthesis and in methylation reactions [8]. $\mathrm{N}_{2} \mathrm{O}$ induced oxidation of vitamin B12 converts vitamin B12 from a reduced to an oxidized form, which inhibits the activity of methionine synthase, leading to impairment of methylation reactions and DNA synthesis. This leads to the accumulation of homocysteine. However, the methylmalonyl-CoA mutase pathway is not affected because this pathway depends on the oxidized form rather than the reduced form of vitamin B12.

Hyperpigmentation of the skin with megaloblastic anemia associated with vitamin B12 deficiency was first observed by Cook in 1944 [9-11]. Pigmentation due to vitamin B12 deficiency can occur at any location on the body, but it occurs most commonly on the hands and feet, particularly in the interphalangeal joints, and sometimes in the fingernails and toenails. The pathogenesis of hyperpigmentation of the skin associated with vitamin B12 deficiency remains unclear. Gilliam and Cox [11] postulated that vitamin B12 deficiency leads to lower intracellular levels of reduced-type glutathione, which has a tyrosinaseinhibiting effect, and that once this effect declines, the epidermal melanocytes are stimulated to produce melanin. Moreover, the decreased reduced-type glutathione concentration 
retards mitosis and reduces DNA synthesis, causing dermal atrophy and hyperpigmentation. However, the reasons underlying the predominant occurrence of pigmentation in specific areas and its occurrence in only certain cases need further investigation.

$\mathrm{N}_{2} \mathrm{O}$-induced neuropathy was initially recognized by Layzer et al. in 1978 [12]. In that study, the common initial symptoms were numbness of the distal limbs and imbalance, complicated by weakness in the lower extremities, with some patients becoming too unsteady to walk without assistance. The numbness sometimes travelled upwards from the feet and also caused clumsiness in the hands. Physical examination of the patients revealed diminished deep tendon reflexes of the lower legs, positive Babinski sign, loss of sensation in the dorsal columns, and a wide-based gait. These neurological manifestations were induced by the interaction of $\mathrm{N}_{2} \mathrm{O}$ with vitamin $\mathrm{B} 12$ and did not resolve until $\mathrm{N}_{2} \mathrm{O}$ exposure was discontinued [12]. $\mathrm{N}_{2} \mathrm{O}$-induced oxidation of vitamin $\mathrm{B} 12$ is irreversible, and recovery of methionine synthase activity requires the reduced form of vitamin B12 to be newly synthesized. After discontinuation of $\mathrm{N}_{2} \mathrm{O}$ exposure and provision of adequate treatment, previous studies have shown that normal enzyme activity recovers within 3-4 days [13].

In the present case, the clinical features of multiple hyperpigmented macular patches on the skin, myeloneuropathy, loss of sensation in the posterior column, poor balance, and pancytopenia were consistent with the diagnosis of vitamin B12 deficiency. Moreover, laboratory studies revealed elevated serum homocysteine levels and polyneuropathy in both lower limbs, which further confirmed the diagnosis of B12 deficiency [14].

The treatment of $\mathrm{N}_{2} \mathrm{O}$ abuse-induced vitamin B12 deficiency is similar to the treatment of pernicious anemia, and comprises 1 of 2 regimens: (1) intramuscular injections of 1,000 $\mu \mathrm{g}$ vitamin B12 (cyanocobalamin) daily for 1 week, followed by weekly injections for 4-8 weeks, and then monthly injections until clinical resolution; or (2) daily oral administration of 1,000-2,000 $\mu \mathrm{g}$ cyanocobalamin until clinical resolution [15].

Our patient, a well-nourished young woman diagnosed with vitamin B12 deficiency, had hyperpigmentation over the dorsa of the fingers, toes, and trunk, associated with pancytopenia and myeloneuropathy. Unlike previously reported cases [10, 11], there were no malabsorption syndromes, nor was there malnutrition or any other gastrointestinal causes. The patient revealed a 2-year history of $\mathrm{N}_{2} \mathrm{O}$ abuse upon further evaluation. The hyperpigmented lesions improved with daily intramuscular injections of $1,000 \mu \mathrm{g}$ cyanocobalamin and cessation of $\mathrm{N}_{2} \mathrm{O}$ abuse. $\mathrm{N}_{2} \mathrm{O}$ abuse may induce vitamin $\mathrm{B} 12$ deficiency, leading to hyperpigmentation of the skin, hair changes, megaloblastic anemia, pancytopenia, subacute combined degeneration, depression, psychosis, and a possible increased risk of myocardial infarction and stroke; however, many of these symptoms, including hyperpigmentation, are reversible with vitamin B12 supplementation.

It is important for clinicians to recognize the manifestations of $\mathrm{N}_{2} \mathrm{O}$ abuse and begin treatment as early as possible. This case report has important diagnostic implications in relation to presentations of skin pigmentation in association with neuropathy. When vitamin B12 deficiency occurs in healthy individuals in the absence of gastrointestinal causes, malnutrition, and malabsorption, $\mathrm{N}_{2} \mathrm{O}$ exposure should be considered in the differential diagnosis. A detailed and complete patient history is crucial for establishing the diagnosis of $\mathrm{N}_{2} \mathrm{O}$ intoxication-induced vitamin B12 deficiency.

\section{Disclosure Statement}

The authors declare that they have no conflicts of financial or personal interest. 
Chiang et al.: Recreational Nitrous Oxide Abuse-Induced Vitamin B12 Deficiency in a

Patient Presenting with Hyperpigmentation of the Skin

\section{References}

1 Oh R, Brown DL: Vitamin b12 deficiency. Am Fam Physician 2003;67:979-986.

-2 Aaron S, Kumar S, Vijayan J, Jacob J, Alexander M, Gnanamuthu C: Clinical and laboratory features and response to treatment in patients presenting with vitamin B12 deficiency-related neurological syndromes. Neurol India 2005;53:55-58, discussion 59.

- 3 Ng J, O'Grady G, Pettit T, Frith R: Nitrous oxide use in first-year students at Auckland University. Lancet 2003;361:1349-1350.

4 Chanarin I: Cobalamins and nitrous oxide: a review. J Clin Pathol 1980;33:909-916.

5 Banks RGS, Henderson RJ, Pratt JM: Reactions of gases in solution. Part III. Some reactions of nitrous oxide with transition-metal complexes. J Chem Soc A 1968:2886-2889.

6 Scott J, Weir D: Folate/vitamin B12 inter-relationships. Essays Biochem 1994;28:63-72.

7 Green R, Miller J: Vitamin B12; in Zempleni J, Rucker RB, McCormick DB, Suttie JW (eds): Handbook of Vitamins, ed 4. Boca Raton, CRC Press, 2007, pp 413-457.

8 Alt RS, Morrissey RP, Gang MA, Hoffman RS, Schaumburg HH: Severe myeloneuropathy from acute highdose nitrous oxide $\left(\mathrm{N}_{2} \mathrm{O}\right)$ abuse. J Emerg Med 2011;41:378-380.

9 Cook B: A vitamin B12 deficiency syndrome allied to sprue. Indian Med Gaz 1944;79:429-437.

10 Baker SJ, Ignatius M, Johnson S, Vaish SK: Hyperpigmentation of skin. A sign of vitamin-B12 deficiency. Br Med J 1963;1:1713-1715.

11 Gilliam JN, Cox AJ: Epidermal changes in vitamin B12 deficiency. Arch Dermatol 1973;107:231-236.

12 Layzer RB, Fishman RA, Schafer JA: Neuropathy following abuse of nitrous oxide. Neurology 1978;28:504506.

13 Nunn JF: Clinical aspects of the interaction between nitrous oxide and vitamin B12. Br J Anaesth 1987;59:313.

14 Hanada K: Vitamin B12 deficiency; in Tamaki K (ed): Comprehensive Handbook of Clinical Dermatology. Tokyo, Nakayama Shoten, 2002, pp 250-251.

-15 Stabler SP: Clinical practice. Vitamin B12 deficiency. N Engl J Med 2013;368:149-160.
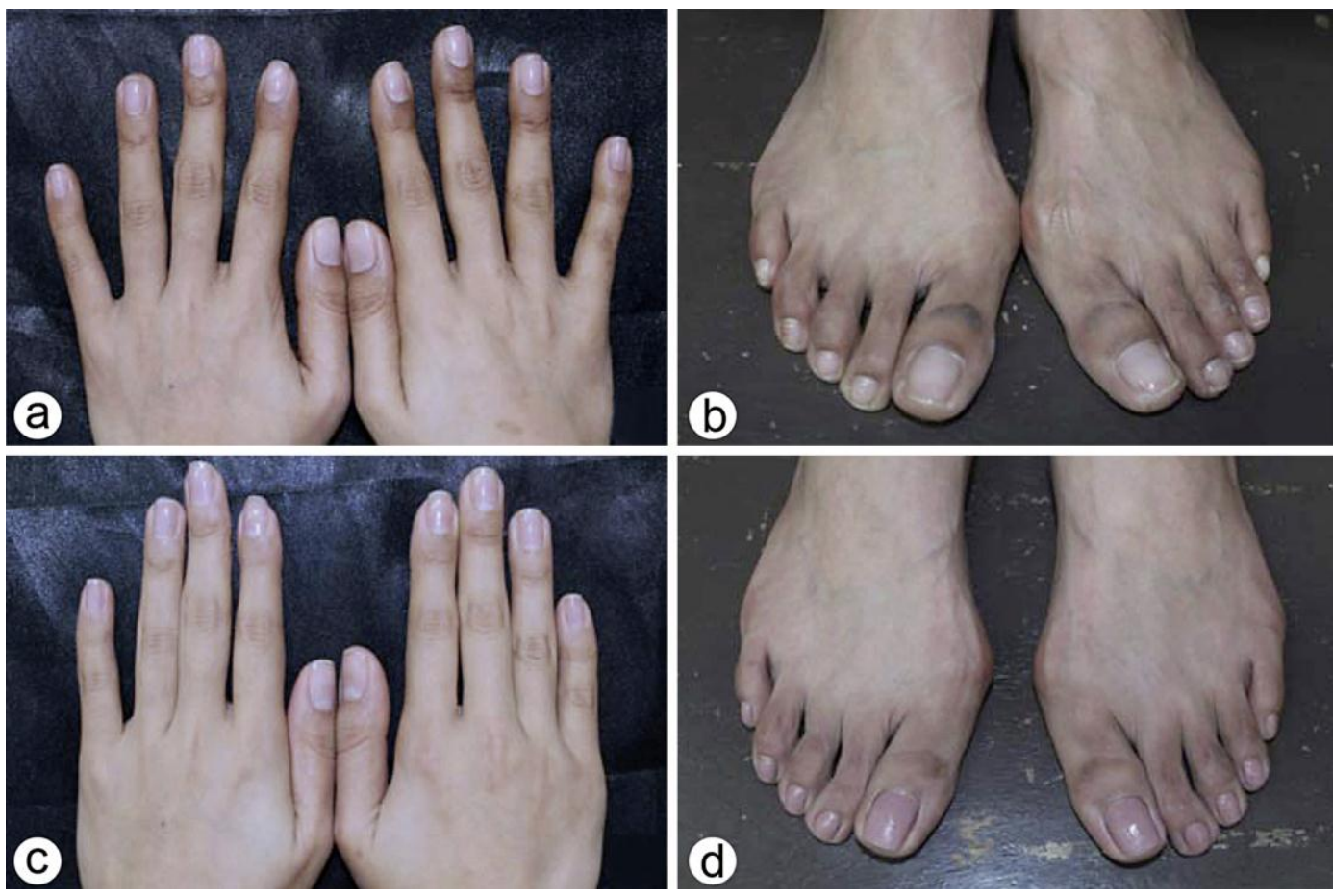

Fig. 1. Hyperpigmentation of both hands and feet. Initially, the patient showed multiple asymptomatic hyperpigmented macular patches over the dorsa of the fingers (a) and toes (b). After about 1 month of vitamin B12 (cyanocobalamin) therapy, the pigmented lesions showed considerable improvement (c, d). 
Chiang et al.: Recreational Nitrous Oxide Abuse-Induced Vitamin B12 Deficiency in a Patient Presenting with Hyperpigmentation of the Skin

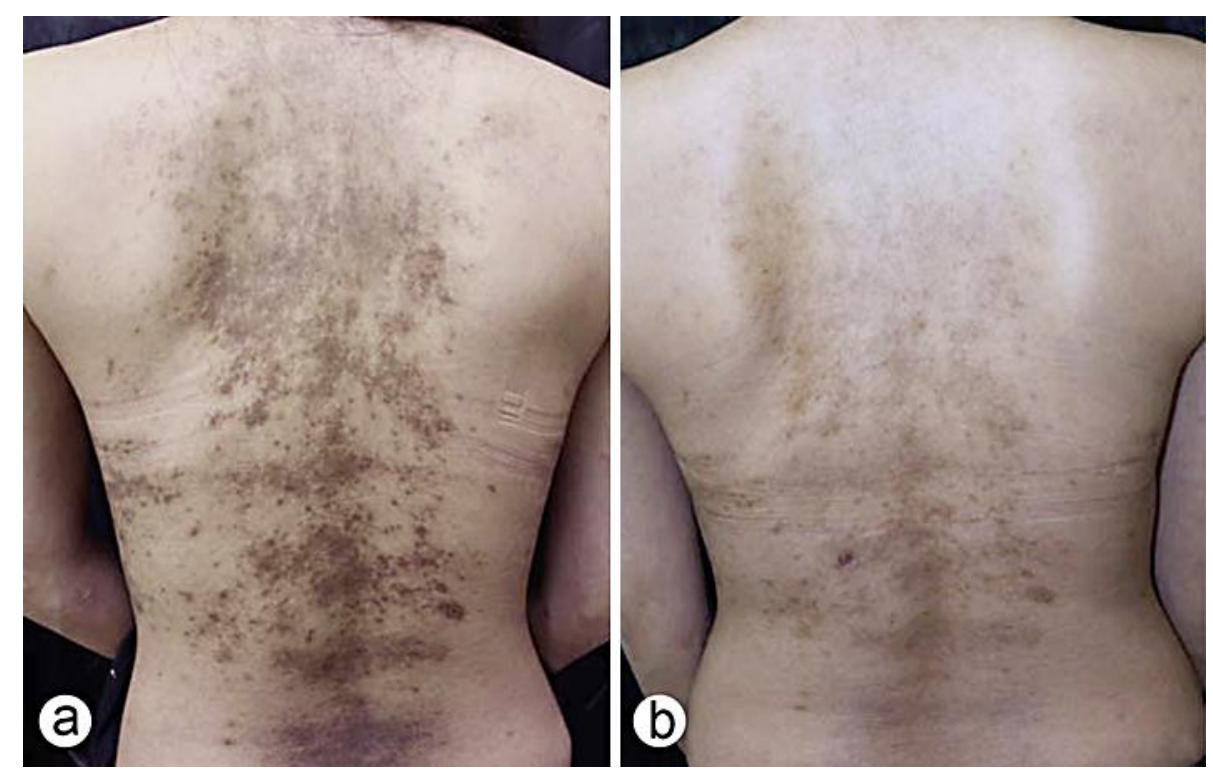

Fig. 2. Hyperpigmented maculopapular lesions on the trunk. Multiple asymptomatic hyperpigmented macular patches over the trunk (a), which improved significantly after 1 month of vitamin B12 (cyanocobalamin) therapy (b). 